\title{
SIGMODONTINOS (RODENTIA, MURIDAE) DEL HOLOCENO INFERIOR DE BOLIVIA
}

\author{
Ulyses F.J. PARDIÑAS' y Carlos A. GALLIARI \\ Departamento Científico Paleontología Vertebrados, Museo de La Platia, \\ Paseo del Bosque s/n. 1900 Lal Plata, CONICET. \\ "Instituto de Limnología "Dr. Raúl Ringuelet". Casilla de Correo 712, 1900 Lal \\ Platti, CONICET. Argentinal.
}

\begin{abstract}
Pardiñas, U.F.J. y Galliari, C.A. 1998. Sigmodontinos (Rodentia, Muridae) del Holoceno inferior de Bolivia. |Early Holocene sigmodontines (Rodentia, Muridae) from Bolivia]. Revista Espainola de Paleontología. 13 (1), 17-25. ISSN ()213-6937.
\end{abstract}

\begin{abstract}
A small sample of fossil muroids found in the Middle Member of the Nuapua Formation (Holocene), outcropping at Quebrada de Nuapua (20 $52^{\prime} \mathrm{S} ; 63^{\circ}$ ()4’ W: $500 \mathrm{~m}$ over sea level). Chuquisaca department, Bolivia, was studied. The remains were assigned to the sigmodontines Holochilus chacarius balnearum (Oryzomyini), Necromys sp. (Akodontini), Calomyss sp., and Graomys sp. (Phyllotini). All of these genera and species are at present distributed in southeast Bolivia. The taxonomy of the genus Holochilus in this area is discussed. The differentiation of $H$. sciureus, $H$. chacarius, and H. brasiliensis is justified through their dental morphology. Modern populations of this genus in southeast Bolivia are referred to $H$. chacarius. Noteworthy, the record of "Zygodontomys" cited by previous authors in the fossil sample of Nuapua, corresponds to the genus Necromys (= Bolomys, partim). The scarce fossil sigmodontines known from Ñupua suggest Chacoan conditions for the lower Holocene similar to the present ones in the area.
\end{abstract}

Keywords: Sigmodontinae, Ñuapua, Bolivia, Holocene, taxonomy.

\section{RESUMEN}

Se estudió una pequeña colección de muroideos (Rodentia, Muridae) fósiles provenientes del Miembro Medio de

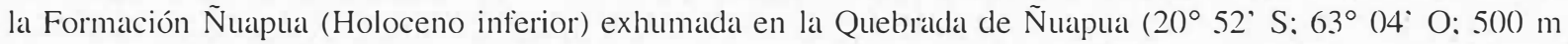
sobre el nivel del mar), departamento Chuquisaca, Bolivia. Se determinaron restos asignables a los sigmodontinos Holochilus chacarius balnearum (Oryzomyini), Necromys sp. (Akodontini), Calomys sp. y Graomyss sp. (Phyllotini), todos géneros y especies actualmente distribuidos en el sudeste boliviano. Se discute la taxonomía del género Holochilus en la región, justificándose a través de rasgos de la morfología dentaria la diferenciación de $H$. sciureus, $H$. chacarius y $H$. brasiliensis. Las poblaciones actuales del género en el sureste de Bolivia se refieren a la especie Holochilus chacarius. Asimismo, se destaca que el registro de "Zygodontomỵs" efectuado por autores previos para la muestra fósil de Nuapua corresponde al género Necromỵs (= Bolomys, parte). Los escasos sigmodontinos fósiles conocidos para Ñuapua sugieren condiciones chaqueñas para el Holoceno inferior, similares a las actuales en el área.

Palabras clave: Sigmodontinae, Ñuapua, Bolivia, Holoceno, taxonomía.

\section{INTRODUCCIÓN}

Los sigmodontinos más antiguos conocidos se registran en el Plioceno Superior (Edad Chapadmalalense inferior; veáse Cione y Tonni, 1995) del suroeste de la provincia de Buenos Aires, Argentina. La mayor parte del registro paleontológico de esta subfamilia proviene de los depósitos del Cenozoico tardío de la región pampeana (Reig, 1987; Pardiñas, 1995a). Unas pocas localidades extra-argentinas han brindado abundante información sobre la diversidad de estas taxocenosis en ambientes tropicales (e.g. cavernas del área de Lagoa Santa, Minas Gerais, Brasil y La Calera, Cachi, Ecuador; véase Winge, 1887; Voss y Myers, 1991; Fejfar et al., 1993).

Para Bolivia se ha destacado la existencia de sigmodontinos fósiles en dos localidades. Hofftstetter (1963) menciona el registro de Holochilus en el Pleistoceno medio-superior de Tarija. Para esta localidad, Reig (en Marshall et al., 1984) cita Kunsia, Oxymycterus, Nectomys, Phyllotis, Andinomyss y Calomys (véase 


\begin{tabular}{|c|c|c|c|c|c|c|c|c|c|c|c|c|c|c|c|c|c|c|c|}
\hline ESPECIE & EJEMPLAR & $\mathrm{m} 1-3$ & Lm1 & Am1 & Lm2 & Am2 & Lm3 & Am3 & DIA & ALM & M1-3 & LM1 & AM1 & LM2 & AM2 & LM3 & AM3 & APZ & EDAD* \\
\hline Holochilus c. balnearum & MLP 95-V-26-1a & 7,85 & 2,96 & 1,84 & 2,20 & 2,12 & 2,48 & 1,88 & 5,04 & 7,55 & & & & & & & & & A \\
\hline Holochilus c. balnearum & MLP 95-V-26-1b & 7,68 & 2,88 & 2,00 & 2,08 & 2,20 & 2,48 & 1,92 & 4,79 & & & & & & & & & & SA \\
\hline Holochilus c. balnearum & MLP 95-V-26-1c & 7,85 & 3,00 & 1,88 & 2,16 & 2,20 & 2,40 & 2,00 & & & & & & & & & & & A \\
\hline Holochilus c. balnearum & MLP 95-V-26-1d & & 2,72 & 1,96 & 2,00 & 2,16 & & & & & & & & & & & & & SA \\
\hline Holochilus c. balnearum & MEDIA & 7,79 & 2,89 & 1,92 & 2,11 & 2,17 & 2,45 & 1,93 & 4,91 & 7,55 & & & & & & & & & \\
\hline Necromys sp. & MLP 95-V-26-2 & 5,62 & 2,00 & 1,32 & 1,56 & 1,36 & 1,28 & 1,04 & & & 5,45 & 2,64 & 1,40 & 1,40 & 1,36 & 0,80 & 1,04 & 2,96 & SA \\
\hline Calomys $s p$. & MLP 95-V-26-3 & & 1,38 & 1,00 & 1,00 & 0,96 & & & & & & & & & & & & & AV \\
\hline Graomys sp. & MLP 95-V-26-4 & & 2,44 & 1,52 & & & & & & & & & & & & & & & A \\
\hline
\end{tabular}

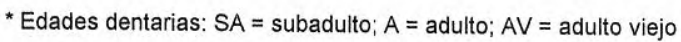

Tabla 1. Medidas cráneo-mandíbulares y dentarias (en milímetros) de los restos de sigmodontinos fósiles de Ñuapua (Bolivia).

también Marshall y Sempere, 1991). Para el Holoceno inferior de Ñuapua, Hoffstetter (1968) indica el hallazgo de Holochilus brasiliensis y Zygodontomys; por su parte Reig (en Marshall et al., 1984) agrega Holochilus, Calomys y Graomys.

Con la excepción del reciente trabajo de Steppan (1996), no existe ninguna descripción publicada sobre los muroideos fósiles de Bolivia y su significación paleoambiental. Esta contribución aporta las primeras descripciones para los sigmodontinos de Ñuapua, sobre la base del estudio de una pequeña colección existente en el Museo de La Plata.

\section{ÑUAPUA: CONTEXTOS GEOGRÁFICO Y ESTRATIGRÁFICO}

La Quebrada de Ñuapua es una de las localidades fosilíferas más ricas de Bolivia. Situada en el sureste de dicho país $\left(20^{\circ} 52^{\prime} \mathrm{S} ; 63^{\circ} 04^{\prime} \mathrm{O} ; 500 \mathrm{~m}\right.$ s.n.m.), departamento de Chuquisaca, fue estudiada por Branisa y Hoffstetter (Hoffstetter, 1968) y, más recientemente por MacFadden y Wolff (1981). Este yacimiento se ubica en el borde occidental de la actual Región Chaqueña, caracterizada climáticamente por una estación seca prolongada, con lluvias estacionales locales y vegetación xerofítica (Hoffstetter, 1968: 824-825).

La sucesión estratigráfica comporta tres miembros que integran la denominada Formación Ñuapua. El Miembro Inferior ha brindado abundante megafauna referible al Pleistoceno Medio-Superior (véase la discusión en Marshall et al., 1984: 32-33). El Miembro Medio es portador de numerosos restos correspondientes a géneros aún vivientes y, en menor medida, a megamamíferos extintos. Dos dataciones radiocarbónicas sobre la fracción de colágeno, $7200 \pm 400$ y $6600 \pm 370$ ar AP (Marshall et al., 1984: 34; Marshall y Sempere, 1991: 643), parecen indicar que los sedimentos representan el Holoceno inferior-medio. Finalmente, el Miembro Superior no ha brindado restos fósiles.

Desde un punto de vista paleoambiental, tanto Hoffstetter (1968) como MacFadden y Wolff (1981) coinciden en señalar que, a juzgar por la composición faunística, el Miembro Medio de la Fm. Nuapua se debió depositar bajo condiciones lacustres o al menos de humedad.

\section{MATERIALES Y MÉTODOS}

Los restos de sigmodontinos estudiados provienen del Miembro Medio de la Fm. Ñuapua, cuyos fósiles están “...bien minéralisés et présentent une couleur noire ou bleu foncé caractéristique" (Hoffstetter, 1968: 830). Fueron coleccionados por L. Branisa quien los depositó, junto a huesos de aves y una mandíbula de un representante de los Tapiridae, en el Museo de La Plata (M. Bond, com. pers.).

En la descripción de las estructuras dentarias se sigue la propuesta terminológica de Reig (1977), a excepción de algunos términos tomados de Hershkovitz (1993). Las medidas fueron calculadas con calibre manual (con precisión de cinco centésimas) y ocular micrométrico adosado a lupa binocular Wild M-5, siendo expresadas en todos los casos en milímetros (para los molares se indican únicamente los largos y anchos máximos y las longitudes de las series yugales son alveolares). Las diferencias métricas de los dientes entre taxones de Holochilus fueron analizadas mediante el test de Kruskal-Wallis.

Abreviaturas utilizadas en el texto, tablas y figuras: M1, $\mathrm{M} 2, \mathrm{M} 3, \mathrm{~m} 1, \mathrm{~m} 2, \mathrm{~m} 3$ : primero, segundo y tercer molar superior e inferior, respectivamente. A: ancho. L: largo. DIA: longitud de la diastema. ALM: altura de la rama mandibular medida bajo el m1. APZ: ancho placa zigomática. MLP: Museo de La Plata. MACN: Museo Argentino de Ciencias Naturales "Bernardino Rivadavia". CEMF: Colección Elio Massoia y familia. MNRJ: Museo Nacional de Río de Janeiro. IADIZA CM: Instituto Argentino para el Estudio de las Zonas Aridas, Colección de Mamíferos.

\section{PALEONTOLOGÍA SISTEMÁTICA Y DESCRIPTIVA}

ORDEN RODENTIA Bodwich, 1821

SUBORDEN MYOMORPHA Miller y Gidley, 1918

Familia Muridae Illiger, 1815

Subfamilia Sigmodontinae Wagner, 1843

Tribu Oryzomyini Vorontzov, 1959 (sensu Voss y Carleton, 1993)

Género Holochilus Brandt, 1835

Holochilus chacarius Thomas, 1906 
Holochilus chacarius balnearum Thomas, 1906 Figs. 1a, 2a, y $3 \mathrm{i}-\mathrm{j}$

Material referido: MLP 95-V-26-1a, hemimandíbula izquierda incompleta con m1-3 e incisivo; MLP 95-V-26-1b, hemimandíbula derecha incompleta con m1-3 e incisivo; MLP 95-V-26-1c, hemimandíbula derecha incompleta con m1-3 y MLP 95-V-26-1d, hemimandíbula izquierda incompleta con m1-2. Medidas: véase la Tabla 1.

\section{Descripción}

La rama mandibular es robusta y comprimida en sentido antero-posterior, con un buen desarrollo del proceso mentoniano, característico del género (Fig. 2a).

En el $\mathrm{m} 1$ la fosétida ántero-interna (fosétida anterior de Hershkovitz, 1993) es grande, con un grueso anillo de esmalte, ocupando buena parte del procíngulo y desplazada claramente hacia el borde labial, tanto que en el ejemplar MLP 95-V-26-1a el borde labial de la fosétida es coalescente con el esmalte externo del diente (Fig. 1a). En otro ejemplar de la muestra fósil, la fosétida está comunicada con un amplio protofléxido. El protofléxido penetra en el plano oclusal, llegando hasta algo más allá de la mitad del diente, lo que sumado a la escasa expresión del metafléxido determina un múrido anterior desplazado lingualmente.

Las áreas de los proto- e hipocónido son subiguales y con moderado desgaste adquieren un contorno subrectangular. Ento- y posterofléxido son transversos y de largo equivalente, determinando ento- y posterolófido subiguales, otorgándole al diente un aspecto cuadrado en la parte posterior.

$\mathrm{El} \mathrm{m} 2$ es subcuadrangular en vista oclusal; con moderado desgaste presenta un patrón trilofodonte (Fig. 1a). Sobre el borde labial, el protofléxido queda indicado por una leve entalladura, mientras que el hipofléxido se orienta transversalmente con su fondo opuesto al entolófido. Meta-, ento- y posterolófido tienen orientación transversal y desarrollo similar, aunque el último está algo acortado en sentido labio-lingual.

El m3 es claramente sigmoide, experimentando una marcada simplificación con el desgaste. El metafléxido se dirige hacia adelante y por su disposición oblicua determina un múrido posterior algo inclinado, pese a la orientación transversa del hipofléxido.

\section{Comparaciones}

Una síntesis de las diferencias y similitudes en la morfología de los molares inferiores en diferentes especies de Holochilus se brinda en la Tabla 2 (véase además la Fig. 3). El test de Kruskal-Wallis indicó diferencias significativas en Lm1-3 $(\mathrm{p}=0,005), \operatorname{Lm} 1(\mathrm{p}=$ 0,004), Am1 $(p=0,04)$ y $\operatorname{Lm} 3(p=0,03)$. En la Fig. 4 puede observarse la similitud métrica entre los ejemplares fósiles de Ñuapua y aquéllos referidos a $H$. chacarius balnearum del noroeste de la Argentina.

\section{Comentarios}

No existe consenso sobre la taxonomía del género Holochilus. Hershkovitz (1955) reconoció dos especies, una de las cuales -Holochilus magnus- ha sido transferida
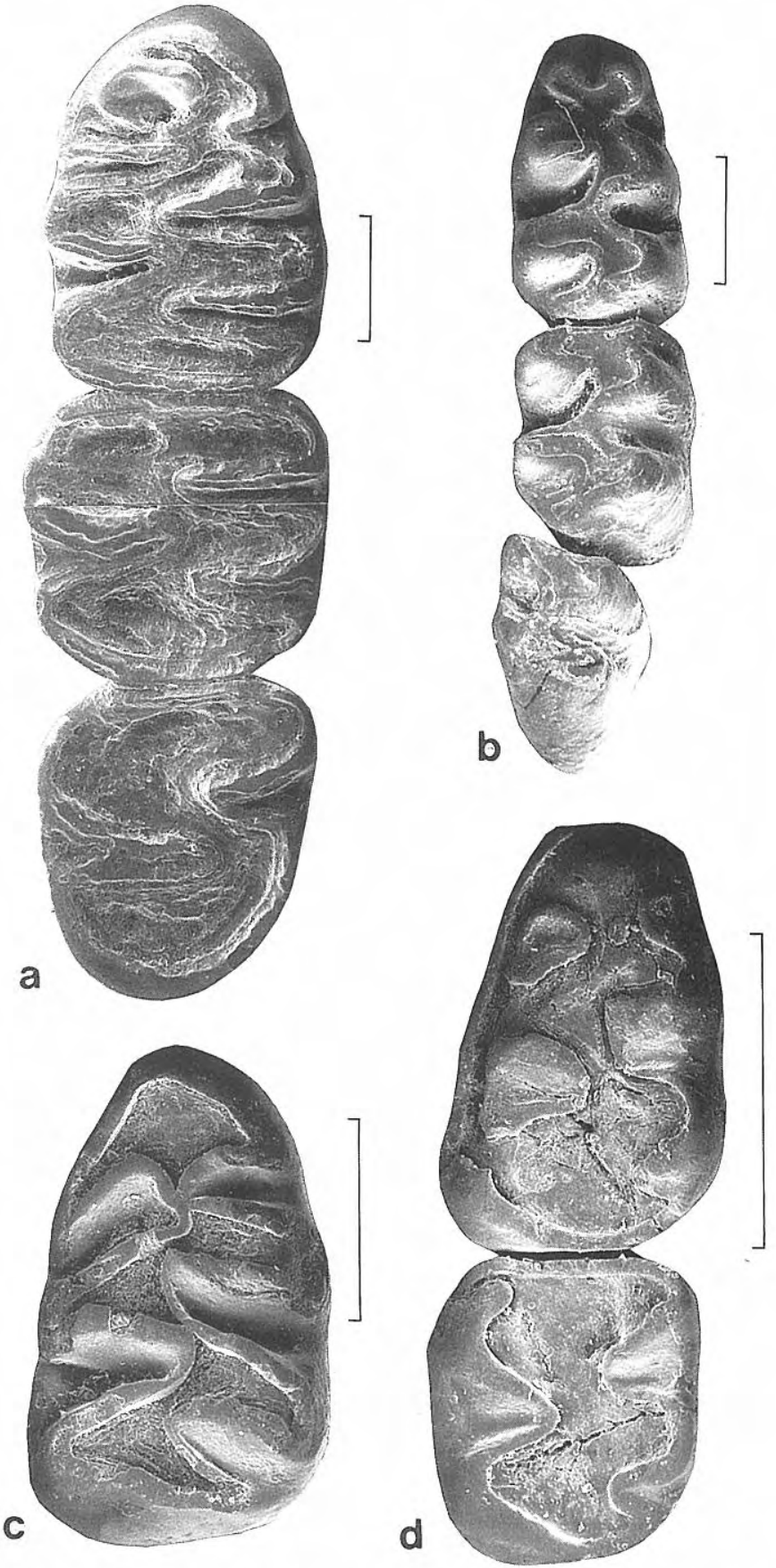

Figura 1. Vistas oclusales de los molares de los sigmodontinos fósiles de Ñuapua (Bolivia): a. Holochilus chacarius balnearum Thomas (MLP 95-V-26-1a), m1-3 izquierdos; b. Necromys sp. (MLP 95-V-26-2), m1-3 derechos; c. Graomys sp. (MLP 95-V-26-4), ml izquierdo; d. Calomys sp. (MLP 95-V-26-3), m1-2 izquierdos. Escala $=1 \mathrm{~mm}$.

a un nuevo género, Lundomys (véase Voss y Carleton, 1993). La otra, H. brasiliensis, incluyó todas las formas nominales descriptas para el género. Massoia (1976), luego de una extensa revisión de especímenes del cono sur suramericano, propuso la existencia de tres grupos: grupo de $H$. sciureus, de $H$. brasiliensis y de H. magnus. Este autor revalidó la especie $H$. chacarius, incluyendo 


\begin{tabular}{|c|c|c|c|c|}
\hline Carácter & $\begin{array}{l}\text { Holochilus } \\
\text { brasiliensis }\end{array}$ & H. c. chacarius & H. sciureus & $\begin{array}{l}\text { H. c. balnearum y } \\
\text { restos de Ñuapua }\end{array}$ \\
\hline $\begin{array}{l}\text { Posición de la } \\
\text { fosétida ántero- } \\
\text { interna del m1 }\end{array}$ & central & $\begin{array}{l}\text { central o algo } \\
\text { volcada labialmente }\end{array}$ & $\begin{array}{l}\text { algo volcada } \\
\text { labialmente }\end{array}$ & volcada labialmente \\
\hline $\begin{array}{l}\text { Forma y tamaño } \\
\text { de la fosétida } \\
\text { ántero-interna del } \\
\text { m1 }\end{array}$ & \begin{tabular}{|l} 
alargada \\
transversalmente, \\
grande
\end{tabular} & $\begin{array}{l}\text { alargada } \\
\text { transversalmente o } \\
\text { subcircular, grande }\end{array}$ & $\begin{array}{l}\text { alargada } \\
\text { transversalmente, } \\
\text { pequeña }\end{array}$ & subcircular, grande \\
\hline $\begin{array}{c}\text { Desarrollo oclusal } \\
\text { del metafléxido } \\
\text { del } \mathrm{m} 1\end{array}$ & $\begin{array}{l}\text { bien desarrollado, } \\
\text { alcanzando la línea } \\
\text { media del diente }\end{array}$ & $\begin{array}{l}\text { poco desarrollado, } \\
\text { no alcanzando la } \\
\text { línea media del } \\
\text { diente }\end{array}$ & $\begin{array}{l}\text { bien desarrollado, } \\
\text { comunicado con el } \\
\text { protofléxido en } \\
\text { subadultos }\end{array}$ & $\begin{array}{l}\text { poco desarrollado, } \\
\text { no alcanzando la } \\
\text { línea media del } \\
\text { diente }\end{array}$ \\
\hline $\begin{array}{c}\text { Proto- e } \\
\text { hipocónido del m1 }\end{array}$ & $\begin{array}{l}\text { áreas de contorno } \\
\text { subtriangular }\end{array}$ & $\begin{array}{l}\text { áreas de contorno } \\
\text { subrectangular }\end{array}$ & $\begin{array}{l}\text { áreas de contorno } \\
\text { subrectangular }\end{array}$ & $\begin{array}{l}\text { áreas de contorno } \\
\text { subrectangular }\end{array}$ \\
\hline $\begin{array}{l}\text { Mesofléxido del } \\
\text { m1 }\end{array}$ & $\begin{array}{l}\text { moderadamente } \\
\text { oblícuo }\end{array}$ & transverso & transverso & transverso \\
\hline $\begin{array}{l}\text { Mesofléxido del } \\
\mathrm{m} 2\end{array}$ & $\begin{array}{l}\text { moderadamente } \\
\text { oblícuo }\end{array}$ & transverso & transverso & transverso \\
\hline $\begin{array}{l}\text { Hipofléxido del } \\
\mathrm{m} 2\end{array}$ & $\begin{array}{l}\text { Hipofléxido más } \\
\text { largo, } \\
\text { sobrepasando la } \\
\text { línea media del } \\
\text { diente }\end{array}$ & $\begin{array}{l}\text { hipofléxido corto, } \\
\text { alcanzando la línea } \\
\text { media del diente }\end{array}$ & $\begin{array}{l}\text { hipofléxido corto, } \\
\text { alcanzando la línea } \\
\text { media del diente }\end{array}$ & $\begin{array}{l}\text { hipofléxido corto, } \\
\text { alcanzando la línea } \\
\text { media del diente }\end{array}$ \\
\hline
\end{tabular}

Tabla 2. Similitudes y diferencias en la dentición inferior entre diferentes representantes del género Holochilus.

dos subespecies: H. c. chacarius, con distribución en la región chaqueña de Argentina y Paraguay y $H$. $c$. balnearum, restringida al noroeste argentino.

Por su parte, Reig (1986) otorgó validez específica a $H$. balnearum, sobre la base de información cariotípica. Revisiones más recientes (e. g., Musser y Carleton, 1993: 704-705) coinciden con Massoia $(1971,1976,1980)$ en la distinción de $H$. chacarius como especie y la inclusión de $H$. balnearum como subespecie de la misma.

La terra typica de $H$. chacarius balnearum se ubica en la provincia de Tucumán -Bañado de San Felipe- y su distribución se extiende desde Jujuy, hasta Catamarca (Cabrera, 1961), incluyendo la provincia de Salta (Mares et al., 1981 -citado como H. brasiliensis).

Para Bolivia únicamente se ha citado $H$. brasilensis amazonicus Osgood, 1915 (Anderson, 1993 y Anderson et al., 1993, siguiendo a Hershkovitz, 1955), aunque más recientemente Anderson (1997) modifica su concepción y asigna los ejemplares a $H$. sciureus amazonicus. Nuestra revisión de los ejemplares del MACN procedentes de Bolivia (véase el Apéndice), asignados a $H$. brasiliensis amazonicus, indica su pertenencia a $H$. chacarius balnearum. No existen, en consecuencia, representantes en el noroeste de Argentina y el sur de Bolivia referibles al grupo de $H$. brasiliensis. Si bien está pendiente la revisión de las especies del género Holochilus de la vertiente andina, siendo uno de los problemas la diferenciación entre $H$. amazonicus y $H$. chacarius, esta última tiene prioridad en caso de ser coespecíficas.

Cabe destacar que Steppan (1996) describe una nueva especie del género Holochilus proveniente del Pleistoceno medio de Tarija, la cual es claramente diferenciable de los restos aquí estudiados por la morfología de sus molares.

Tribu Akodontini Vorontzov, 1959

Género Necromys Ameghino, 1889

\section{Necromys sp.}

Figs. $1 \mathrm{~b}$ y $2 \mathrm{~b}-\mathrm{c}$

Material referido: MLP 95-V-26-2, fragmento anterior de cráneo conservando ambas series yugales y hemimandíbula derecha con m1-3; ambos restos corresponden a un mismo individuo. Medidas: véase la Tabla 1. 


\section{Descripción}

El cráneo está deformado, con los huesos fragmentados, lo cual imposibilita una adecuada descripción, especialmente a nivel del techo craneano (Figs. 2b-c). Los incisivos superiores son moderadamente robustos, la base de los forámenes incisivos alcanza el nivel del protocono (Fig. 2b). La placa zigomática es ancha en sentido anteroposterior, marcadamente excavada en la región de la inserción de la parte profunda del músculo masetero lateral. Su borde libre superior presenta un bisel característico (Fig. 2c).

La mandíbula es robusta. La proyección capsular forma un abultamiento bien notable, que se recuesta mayormente sobre el cóndilo.

El ml conserva un fléxido anteromediano moderadamente desarrollado. Por su parte, el m3 está claramente comprimido en sentido antero-posterior (Fig. 1b). Los molares superiores presentan una corona levemente crestada y las cúspides opuestas. En el M1 el procíngulo muestra la indentación del flexo anteromediano.

\section{Comentarios}

Los caracteres mandibulares distinguen el género Necromys de las especies de Akodon que habitan actualmente en la región. En las especies del grupo de Akodon varius (sensu Myers, 1989), la proyección capsular del incisivo inferior está generalmente menos expresada y ocupa una posición medial, entre el cóndilo y el proceso coronoides.

El género Necromys fue revalidado por Massoia y Pardiñas (1993), incluyendo los sinónimos posteriores Bolomys Thomas, 1916 y Cabreramys Massoia y Fornes, 1967. Para Bolivia, Anderson y Olds (1989) y Anderson (1997) han citado las siguientes especies de este género: $N$. amoenus (Thomas, 1900), N. lactens (Thomas, 1918) y $N$. lenguarum (Thomas, 1898). Según estos autores, en el sur de Bolivia habitan las tres especies, aunque son más frecuentes y abundantes las dos últimas. El resto fósil aquí estudiado puede ser diferenciado de $N$. amoenus por su mayor tamaño, coincidiendo con el rango de $N$. lactens y $N$. lenguarum. Los caracteres craneanos que distinguen estas especies no pueden apreciarse dada la naturaleza fragmentaria del resto fósil. Aún así, las medidas de los molares del resto fósil de Nuapua son más cercanas a las conocidas para $N$. lenguarum, especie propia de la Región Chaqueña (véase Anderson y Olds, 1989).

Tribu Phyllotini Vorontzov, 1959

Género Calomys Waterhouse, 1837

\section{Calomys sp.}

Fig. 1d

Material referido: MLP 95-V-26-3, fragmento de hemimandíbula izquierda con $\mathrm{m} 1$ y $\mathrm{m} 2$. Medidas: véase la Tabla 1.

\section{Descripción}

Los molares son claramente crestados y las cúspides apenas alternas. El m1 presenta una destrucción del esmalte
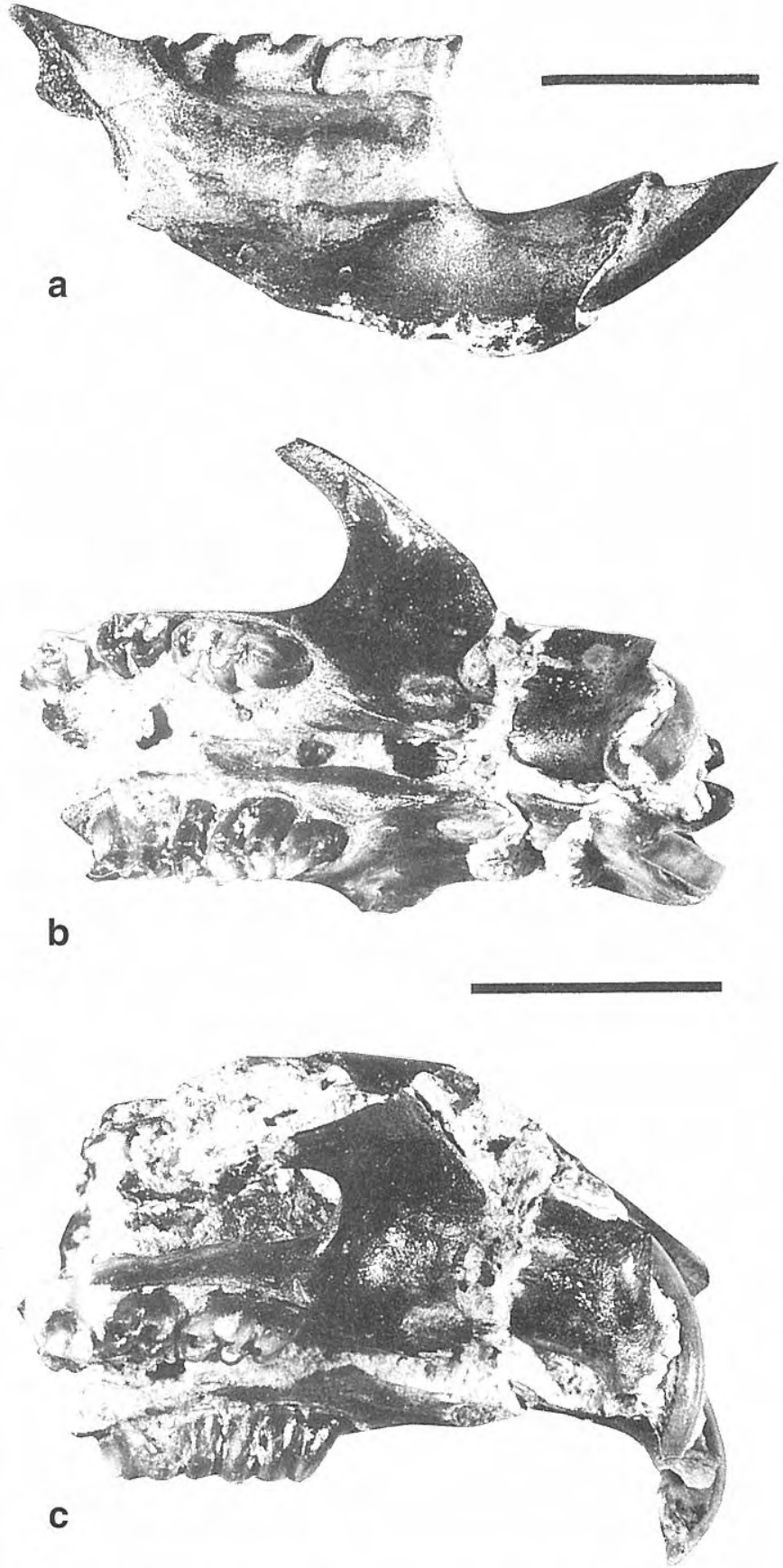

Figura 2. a. Holochilus chacarius balnearum Thomas (MLP 95-V-26-1a), hemimandíbula derecha en vista labial; b y c. Necromys sp. (MLP 95-V-262), fragmento anterior de cráneo en vista ventral y lateral, respectivamente. Escala $=5 \mathrm{~mm}$.

perimetral, lo cual dificulta su descripción y comparación (Fig. 1d). A las dos raíces usuales se le agregan dos accesorias, lingual y labial, lo que constituye un carácter frecuente en las especies de Calomys. El m2 presenta un cíngulo anterolabial que continúa en forma de un cordón sobre el borde labial del diente. El alvéolo del $\mathrm{m} 3$ es trilocular.

\section{Comentarios}

El resto de Ñuapua, por la coincidencia de sus 

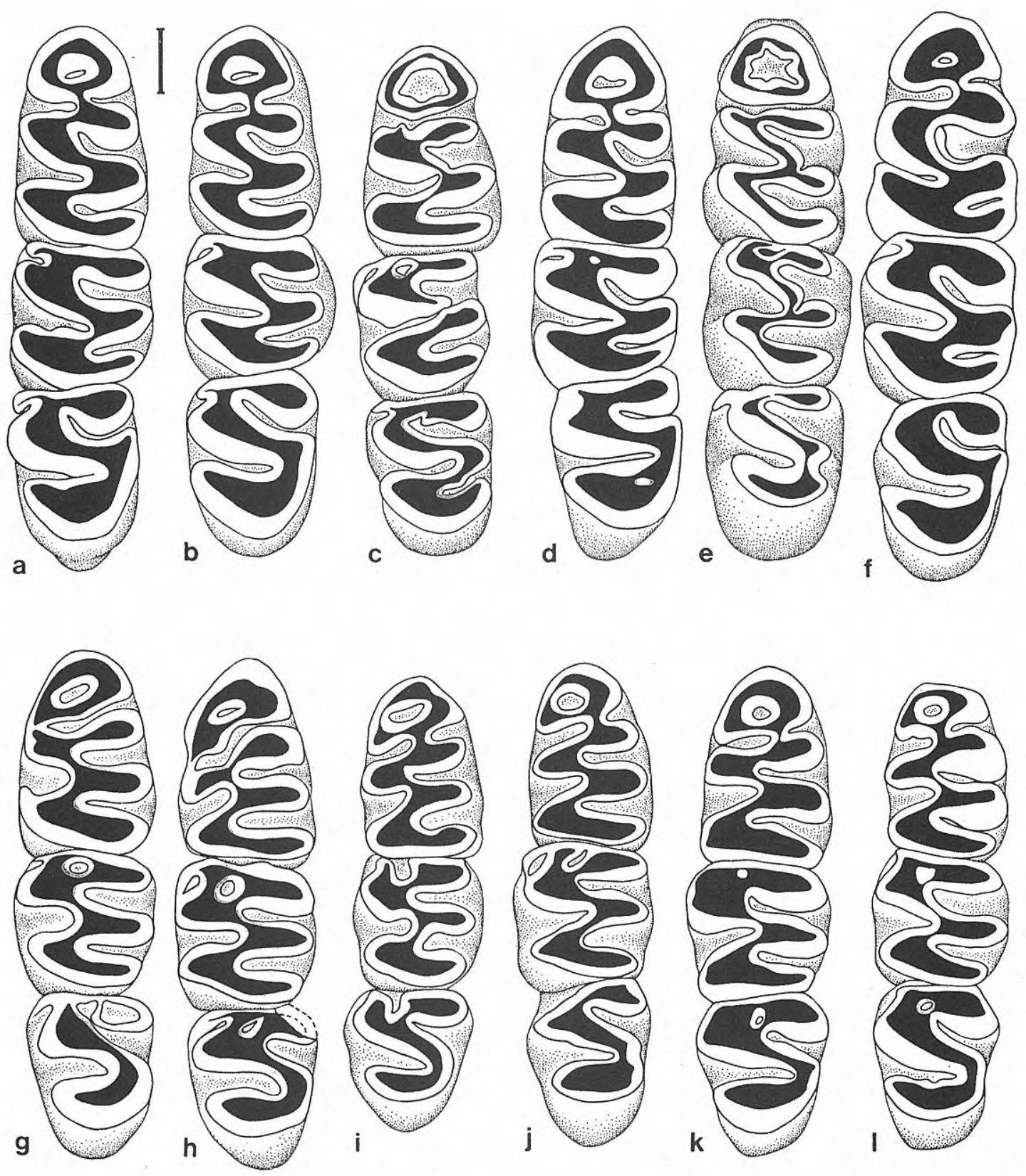

Figura 3. Molares inferiores (vista oclusal) en diferentes representantes del género Holochilus: a y b. Holochilus brasiliensis darwini (CEMF 3672 y 888, respectivamente); c y d. H. b. vulpinus (CEMF 3683 y CAF 1042); e y f. H. b. brasiliensis (MNRJ 4097 y 4088); g y h. H. sciureus (MNRJ 4209 y 4207); i y j. H. chacarius balnearum $($ CEMF s/n); $\mathrm{k}$ y l. H. c. chacarius $($ CEMF 3424 y 3421$)$. Escala $=1 \mathrm{~mm}$.

medidas, podría ser referible al grupo de especies de tamaño pequeño del género Calomys, por lo que pueden excluirse de esta discusión C. boliviae (Thomas, 1901), C. callidus (Thomas, 1916) y C. callosus (Rengger, 1830). Dentro de las especies de tamaño pequeño presentes actualmente en la región -C. laucha (Fischer, 1814), C. lepidus (Thomas, 1884) y C. musculinus (Thomas, 1913)- la distinción con restos fragmentarios es sumamente compleja (véase la discusión en Pardiñas y Lezcano, 1995), por lo cual optamos por la determinación abierta aquí propuesta. Por otro lado, el alvéolo del m3 presenta tres loci, siendo ésta una condición usual en Calomys laucha y C. musculinus, diferente de las especies del género Eligmodontia, donde sólo se la observa ocasionalmente.

Género Graomys Thomas, 1916

Graomys sp.

Fig. 1c

Material referido: MLP 95-V-26-4, fragmento de hemimandíbula izquierda con m1. Medidas: véase la Tabla 1. Descripción

La rama mandibular se encuentra muy fragmentada. El 
$\mathrm{m} 1$ es de corona plana y con cúspides alternas. El procíngulo está comprimido en sentido antero-posterior y se continúa hacia atrás por un extenso cíngulo anterolabial. Las áreas de los proto- e hipocónido son subiguales y subtriangulares. El posterofléxido se conserva con un desarrollo moderado (Fig. 1c). Sobre el borde lingual se destaca una raíz accesoria de posición media.

\section{Comentarios}

La distinción entre Phyllotis y Graomys con restos fragmentarios no es una tarea sencilla (véase la discusión en Pardiñas y Deschamps, 1996: 374). Generalmente, en las especies de Phyllotis, el $\mathrm{m} 1$ muestra una temprana obliteración del protofléxido, con la consecuente formación de una protofosétida, mientras que en Graomys este fléxido permanece abierto aún en ejemplares adultos. Además, en este último, los juveniles presentan una parcial desvinculación del procíngulo del $\mathrm{m} 1$ con respecto a las demás estructuras superficiales del molar (véase Pardiñas, 1995b). Ambos rasgos caracterizan al resto fósil de Nuapua y permiten referirlo al género Graomys. Un problema distinto es su asignación a alguna de las especies vivientes en el área (véase Anderson, 1997): G. pearsoni Myers, 1977, G. griseoflavus (Waterhouse, 1837) o G. domorum (Thomas, 1902). Los m1 de G. pearsoni son fácilmente distinguibles por su marcada alternancia de cúspides y procíngulo romboidal con fosétida anteromediana persistente. G. griseoflavus y $G$. domorum muestran denticiones muy similares, razón que impide una determinación precisa hasta contar con restos más completos.

\section{DISCUSIÓN Y CONCLUSIONES}

\section{Aspectos tafonómicos}

Poco es lo que puede decirse sobre la historia tafonómica del agregado de Ñuapua a partir de la pequeña muestra estudiada. Sin embargo, los restos presentan un leve redondeamiento de sus bordes de fractura y estrías superficiales. Tales características parecen indicar un transporte moderado en condiciones subacuáticas previo al enterramiento. Por otra parte, en tres especímenes se han observado signos de corrosión (disolución del esmalte perimetral) como los señalados por Andrews (1990) para los restos provenientes de agregados formados por aves rapaces.

Cabe destacar que Hoffstetter (1968) indica que los restos de megamamíferos recuperados en el Miembro Medio de la Fm. Ñuapua presentan evidentes signos de transporte y podrían provenir, en realidad, del Miembro Inferior. En oposición y como se ha expresado precedentemente, los restos de sigmodontinos indican sólo un transporte moderado, siendo posiblemente parautóctonos.

\section{Aspectos bioestratigráficos}

Todos los géneros registrados en la muestra fósil de Nuapua también están presentes en el Plio-Pleistoceno de

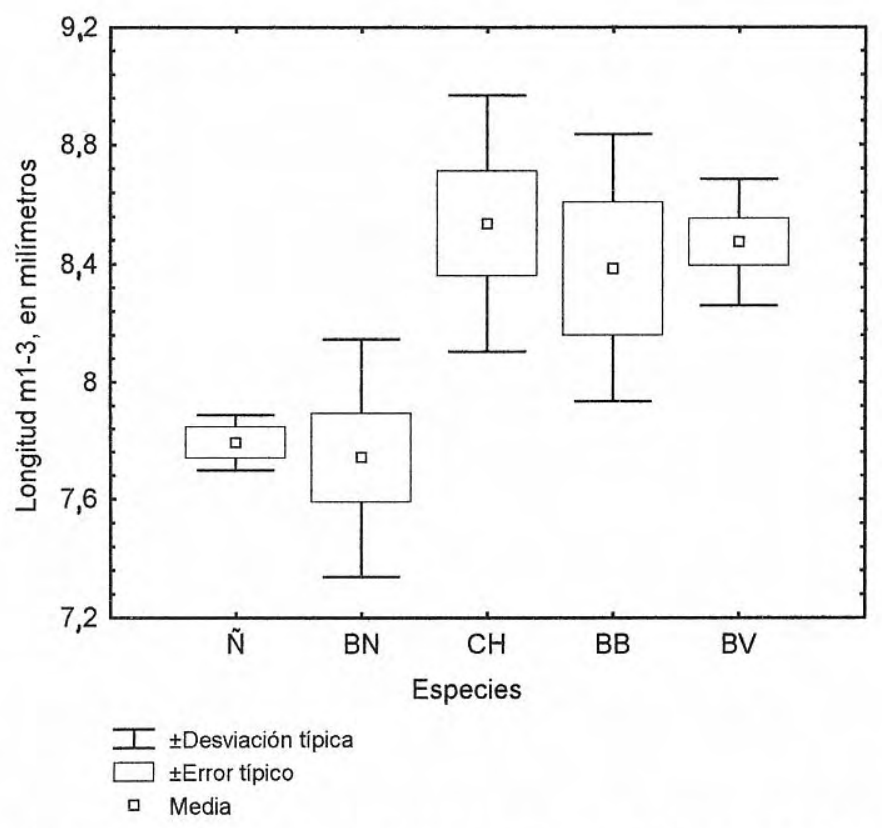

Figura 4. Media, desviación y error estándar de la longitud de la serie molar inferior en muestras de Holochilus. $\tilde{N}$ : ejemplares fósiles de Nuapua, BN: $H$. chacarius balnearum, $\mathrm{CH}: H$. c. chacarius, BB: H. b. brasiliensis, BV: $H$. brasiliensis vulpinus. Test de Kruskall Wallis: $\mathrm{p}=0,005$.

la región pampeana de Argentina. Para Necromys y Graomys fueron descritas especies extintas en sedimentos referidos al Plioceno Medio-Superior de la provincia de Buenos Aires (véase Reig, 1978). Tanto Calomys como Holochilus se registran por primera vez en el Pleistoceno Inferior-Medio (Piso Ensenadense) de diversas localidades de la provincia de Buenos Aires (véase Pardiñas, 1995a; Pardiñas y Deschamps, 1996).

Para Bolivia, Steppan (1996) describe una nueva especie extinta referida al género Holochilus, proveniente del Ensenadense de la cuenca de Tarija. Para esta misma localidad, Reig (en Marshall et al., 1984) menciona los géneros Andinomys, Calomys, Kunsia, Nectomys, Oxymycterus y Phyllotis. Hoffstetter (1968) y Marshall y Sempere (1991) mencionan la presencia de Zygodontomys, que muy posiblemente corresponde al taxón aquí referido como Necromys (véase Voss, 1991 para una discusión sobre la extensión de ambos géneros).

A nivel específico, el registro fósil de Holochilus chacarius balnearum es el primero conocido para América del Sur, de lo que se desprende que los procesos de especiación y adaptativos que dieron origen a algunos sigmodontinos vivientes son, por lo menos, previos al Holoceno temprano.

\section{Aspectos paleoambientales}

Un aspecto destacable de la paleofauna de sigmodontinos de Nuapua es que todos los componentes registrados habitan actualmente en el área o en regiones cercanas (véase Anderson, 1997). Holochilus chacarius balnearum, Graomys griseoflavus, Necromys lenguarum, 
Calomys laucha, Akodon toba y Oligoryzomys chacoensis son algunos elementos frecuentes en la Región Chaqueña occidental (véase Massoia, 1971; Anderson, 1997). Por otra parte, las dos únicas muestras hasta ahora conocidas para la localidad -la estudiada por Hoffstetter (1968) y luego por Reig (en Marshall et al., 1984) y la aquí presentada- contienen los mismos roedores: Holochilus, Necromys, Calomys y Graomys. Esto permite inferir, con las limitaciones inherentes a muestras reducidas y con historias tafonómicas poco conocidas, la existencia durante el Holoceno inferior de ambientes chaqueños similares a los registrados actualmente.

Aún así, es importante recalcar que un muestreo detallado de los sigmodontinos fósiles de Nuapua puede contribuir en el futuro a contrastar los modelos paleoclimáticos propuestos por diversos investigadores sobre la base de evidencias principalmente estratigráficas (Servant et al., 1981; Servant et al., 1989).

\section{AGRADECIMIENTOS}

Mariano Bond nos comentó la existencia de los restos fósiles de Nuapua en las colecciones del Museo de La Plata. Martha Piantanida y Olga Vaccaro facilitaron el acceso a las colecciones de sigmodontinos del MACN. Elio Massoia puso a nuestra disposición material inédito sobre Holochilus así como también su vasto conocimiento del género. El Consejo Nacional de Investigaciones Científicas y Técnicas financió este trabajo. A todas las personas e instituciones mencionadas, el especial reconocimiento de los autores.

\section{BIBLIOGRAFÍA}

Anderson, S. 1993. Los mamíferos bolivianos: notas de distribución y claves de identificación. Publicación especial del Instituto de Ecología, Colección Boliviana de Fauna, La Paz, Bolivia, 159 pp.

Anderson, S. 1997. Mammals of Bolivia, taxonomy and distribution. Bulletin of the American Museum of Natural History, 231, 1-652.

Anderson, S. and Olds, N. 1989. Notes on Bolivian mammals. 5. Taxonomy and distribution of Bolomys (Muridae, Rodentia). American Museum Novitates, 2935, 1-22.

Anderson, S., Riddle, B. R., Yates, T. L., y Cook, J. A. 1993. Los mamíferos del Parque Nacional Amboró y la región de Santa Cruz de la Sierra, Bolivia. Special Publication, The Museum of Southwestern Biology, 2, 158.

Andrews, P. 1990. Owls, caves and fossils. British Museum (Natural History), London, 231 pp.

Cabrera, A. 1961. Catálogo de los Mamíferos de América del Sur. Parte II. Revista del Museo Argentino de Ciencias Naturales "Bernardino Rivadavia", Zoología, $4(2), 309-732$.

Cione, A. L. and Tonni, E. P. 1995. Chronostratigraphy and "land-mammal ages" in the Cenozoic of southern South America: principles, practices, and the "Uquian" problem. Journal of Paleontology, 69, 135-159.
Fejfar, O., Blasetti, A., Calderoni, G., Coltorti, M., Ficcarelli, G., Masini, F., Rook, L. and Torre, D. 1993. New finds of cricetids (Mammalia, Rodentia) from the late PleistoceneHolocene of Northern Ecuador. Document Laboratoire Géologique de Lyon, 125, 151-167.

Hershkovitz, P. 1955. South American marsh rats genus Holochilus, with a summary of sigmodont rodents. Fieldiana, Zoology, 37, 639-673.

Hershkovitz, P. 1993. A new central brazilian genus and species of sigmodontine rodent (Sigmodontinae) transitional between akodonts and oryzomyines with a discussion of muroid molar morphology and evolution. Fieldiana, Zoology, New series, 75, 1-18.

Hoffstetter, R. 1963. La faune pléistocéne de Tarija (Bolivie). Note préliminaire. Bulletin du Muséum National d'Histoire Naturelle, 2e Série 35 (2), 194-203.

Hoffstetter, R. 1968. Ñuapua, un gisement de vertébrés pléistocènes dans le Chaco Bolivien. Bulletin du Muséum Nationalle d'Histoire Naturelle, 2e Série 40 (4), 823836.

MacFadden, B.J. and Wolff, R.G. 1981. Geological investigations of Late Cenozoic vertebrate-bearing deposits in southern Bolivia. In: Anais do II Congresso Latino-Americano de Paleontología (Ed. I.T. Sanguinetti), Porto Alegre, Brasil, 2, 765-778.

Mares, M.A., Ojeda R.A. and Kosco, M.P. 1981. Observations on the distribution and ecology of the mammals of Salta Province, Argentina. Annals of the Carnegie Museum, 50, 151-206.

Marshall, L.G. and Sempere, T. 1991. The Eocene to Pleistocene vertebrates of Bolivia and their stratigraphic context: a review. In: Fósiles y Facies de Bolivia. Vol. IVertebrados (Ed. R. Suaréz-Soruco). Revista Técnica de Yacimientos Petrolíferos Fiscales Bolivianos, 12 (3-4), 631-652.

Marshall, L., Berta, A., Hoffstetter, R., Pascual, R., Reig, O., Bombín, M. and Mones, A. 1984. Mammals and stratigraphy: geochronology of the continental mammalbearing Quaternary of South America. Paleovertebrata, Mémoire Extraordinaire, 1-76.

Massoia, E. 1971. Caracteres y rasgos bioecológicos de Holochilus brasiliensis chacarius Thomas ("rata nutria") de la provincia de Formosa y comparaciones con Holochilus brasiliensis vulpinus (Brants) (MammaliaRodentia-Cricetidae). Revista de Investigaciones Agropecuarias, serie 1, Biología y Producción Animal, 8 (1), 13-40.

Massoia, E. 1976. Mammalia. In: Fauna de Agua Dulce de la República Argentina (Dir. R. Ringuelet). Fundación Editorial Ciencia y Cultura, Fascículo 44, 128 pp.

Massoia, E. 1980. El estado sistemático de cuatro especies de cricétidos sudamericanos y comentarios sobre otras especies congenéricas (Mammalia, Rodentia). Ameghiniana, 17 (3), 280-287.

Massoia, E. y Pardiñas, U. F. J. 1993. El estado sistemático de algunos muroideos estudiados por Ameghino en 1889. Revalidación del género Necromys (Mammalia, Rodentia, Cricetidae). Ameghiniana, 30 (4), 407-418.

Musser, G. G. and Carleton, M. D. 1993. Family Muridae. In: Mammal species of the World. A taxonomic and 
geographic reference (Eds. D. E. Wilson and D. A. M. Reeder). Segunda edición, Smithsonian Institution Press, Washington, $1001 \mathrm{pp}$.

Myers, P. 1989. A preliminary revision of the varius group of Akodon (A. dayi, dolores, molinae, neocenus, simulator, toba and varius). Advances in Neotropical Mammalogy (Eds. K. Redford and J.F. Eisenberg). Sandhill Crane Press, Gainesville, Florida, 614 pp.

Pardiñas, U.F.J. 1995a. Capítulo 11. Los roedores cricétidos. In: Evolución climática y biológica de los últimos cinco millones de años en la región pampeana. Un ensayo de correlación con el Mediterráneo Occidental (Eds. M.T. Alberdi, G. Leone y E.P. Tonni). Monografías del Museo Nacional de Ciencias Naturales, CSIC, Madrid, 425 pp.

Pardiñas, U. F. J. 1995b. Sobre las vicisitudes de los géneros Bothriomys Ameghino, 1889, Euneomys Coues, 1874 y Graomys Thomas, 1916 (Mammalia, Rodentia, Cricetidae). Ameghiniana, 32 (2), 173-180.

Pardiñas, U. F. J. y Deschamps, C. 1996. Sigmodontinos (Mammalia, Rodentia) pleistocénicos del sudoeste de la provincia de Buenos Aires (Argentina): aspectos sistemáticos, paleozoogeográficos y paleoambientales. Estudios Geológicos, 52, 367-379.

Pardiñas, U. F. J. y Lezcano, M. J. 1995. Cricétidos (Mammalia, Rodentia) del Pleistoceno tardío del nordeste de la provincia de Buenos Aires (Argentina). Aspectos sistemáticos y paleoambientales. Ameghiniana, 32 (3), 249-265.

Reig, O. A. 1977. A proposed unified nomenclature for the enamelled components of the molar teeth of the Cricetidae (Rodentia). Journal of Zoology (London), 181, 227-241.

Reig, O. A. 1978. Roedores cricétidos del Plioceno supe-rior de la Provincia de Buenos Aires (Argentina). Publicaciones del Museo Municipal de Ciencias Naturales de Mar del Plata "Lorenzo Scaglia”, 2 (8), 164-190.

Reig, O. A. 1986. Diversity patterns and differentiation of high Andean rodents. In: High altitude tropical biogeography (Eds. F. Vuilleumier and M. Monasterio), Oxford University Press, New York, 649 pp.

Reig, O. A. 1987. An assessment of the systematics and evolution of the Akodontini, with the description of new fossil species of Akodon (Cricetidae: Sigmodontinae). Fieldiana, Zoology, New series, 39, 347-399.

Servant, M., Fontes, J., Rieu, M. et Saliège, J. 1981. Phases climatiques arides holocènes dans le sud-ouest de l'Amazonie (Bolivie). Comptes Rendus Académie des Sciences de Paris, Série II, 292, 1295-1297.

Servant, M., Fournier, M., Soubies, F., Suguio, K. et Turcq, B. 1989. Sécheresse holocène au Brésil (18-20 latitude Sud). Implications paléométéorologiques. Comptes Rendus Académie des Sciences de Paris, Série II, 309, 153-156.

Steppan, S. J. 1996. A new species of Holochilus (Rodentia: Sigmodontinae) from the middle Pleistocene of Bolivia and its phylogenetic significance. Journal of Vertebrate Paleontology, 16, 522-530.
Voss, R. S. 1991. An introduction to the Neotropical muroid rodent genus Zygodontomys. Bulletin of the American Museum of Natural History, 210, 1-113.

Voss, R. S. and Carleton, M. D. 1993. A new genus for Hesperomys molitor Winge and Holochilus magnus Hershkovitz (Mammalia, Muridae) with an analysis of its phylogenetic relationships. American Museum Novitates, 3085, 1-39.

Voss, R. and Myers, P. 1991. Pseudoryzomys simplex (Rodentia: Muridae) and the significance of Lund's Collections from the Caves of Lagoa Santa, Brazil. Bulletin of the American Museum of Natural History, 206, 414-432.

Winge, H. 1887. Jordfundne og nulevende Gnavere (Rodentia) fra Lagoa Santa, Minas Geraes, Brasilien. E Museo Lundii, 1 (3), 1-178.

Manuscrito recibido: 27 de noviembre, 1996 Manuscrito aceptado: 30 de julio, 1997

\section{APÉNDICE}

Lista de los ejemplares utilizados como material comparativo (se indica especie, repositorio y localidad).

Holochilus chacarius balnearum: MACN 33.24, Tabacal, Salta, Argentina; MACN 17873, O de Agua Blanca, Tabacal, Salta, Argentina; MACN 13106, Uruma, Santa Cruz, Bolivia; MACN 50.378, río Mamoré, Beni, Bolivia; MACN 33.169, "Jujuy", Argentina; MLP 996-003-5, Escuela Agrotécnica "Miguel M. de Güemes, $5 \mathrm{Km}$ al S de Salta, Salta, Argentina.

Holochilus chacarius chacarius: CEMF 3425, 3426, 3424, 3421, 3422, 3398, Finca Yacaré, Laguna Blanca, Formosa, Argentina.

Holochilus brasiliensis vulpinus: MLP 996-007-1 (lote), Berisso, Buenos Aires, Argentina; MLP 996-008-1 (lote), Pueblo Arrúa, Entre Ríos, Argentina.

Holochilus brasiliensis brasiliensis: MLP 996-009-1 (lote), Loreto, Misiones, Argentina.

Necromys sp.: MLP 996-003-1 (lote), Escuela Agrotécnica "Miguel M. de Güemes", $5 \mathrm{Km}$ al S de Salta, Salta, Argentina; MACN 17569, MACN 17572, MACN 17573, Campo Quijano, Salta, Argentina.

Graomys griseoflavus: IADIZA CM 3788 a 3796, Chamical, La Rioja, Argentina.

Graomys domorum: IADIZA CM 3760, El Cadillal, Tucumán, Argentina.

Phyllotis xanthopygus: MACN 29.268 y MACN 29.259, Aconquija, Tucumán, Argentina; MACN 26.145, cerro San Javier, Tucumán, Argentina, MACN 29.259, "Tucumán” (como "P. darwini tucumanus"); MACN 19401, Laguna Pozuelos, Jujuy, Argentina; MACN 19526, Cerro Hermoso, Jujuy, Argentina.

Phyllotis osilae: MACN 29.264 y MACN 29.267, Aconquija, Tucumán, Argentina.

Calomys cf. C. laucha-C. musculinus: MLP 996-005-1 (lote), Laguna Pozuelos, Jujuy, Argentina; MLP 996-003-10 (lote), Escuela Agrotécnica "Miguel M. de Güemes", 5 Km al S de Salta, Salta, Argentina. 